\title{
Capacidade de retenção de água do dossel vegetativo: comparação entre Mata Atlântica e plantação florestal de eucalipto
}

\author{
Canopy water retention capacity: comparison between the Atlantic Rainforest \\ and the forest plantation of eucalypt
}

\author{
Juliano Daniel Groppo ${ }^{\mathrm{I}}$, Luiz Felippe Salemi ${ }^{\mathrm{II}}$, Jorge Marcos Moraes ${ }^{\mathrm{III}}$, Rodrigo Trevisan ${ }^{\mathrm{IV}}$, \\ Gustavo Bicci Seghesi ${ }^{\mathrm{IV}}$, Luiz Antônio Martinelliv
}

\begin{abstract}
Resumo
A Mata Atlântica, um dos ecossistemas mais ameaçados do planeta, reduzida atualmente a menos de $8 \%$ de sua cobertura original, necessita de atenção especial para sua melhor conservação. O objetivo do estudo foi comparar a capacidade de retenção de água do dossel vegetativo em uma microbacia coberta por Floresta Ombrófila Densa (Mata Atlântica), situada no Parque Estadual da Serra do Mar e outra com cobertura de Eucalyptus urophylla x Eucalyptus grandis em área próxima ao Parque. O monitoramento foi realizado nos anos de 2008 e 2009 através de pluviômetros e a retenção de água do dossel florestal foi avaliada a partir de regressões lineares entre a precipitação (PR) e a precipitação interna (TR). Os resultados mostraram que com exceção da interceptação na época "seca" que apresentou os menores coeficientes de determinação $\left(\mathrm{R}^{2}\right)$, sendo de $77 \%$ no eucalipto e $82 \%$ na Mata Atlântica, as variáveis apresentaram bom ajuste com a precipitação incidente com coeficientes maiores de 90\%. A microbacia de Mata Atlântica obteve maior capacidade de retenção de água das copas em relação ao eucalipto, em todo o período de estudo foi observado que precipitações inferiores a $5 \mathrm{~mm}$ na Mata Atlântica e $0,78 \mathrm{~mm}$ no Eucalipto são totalmente interceptadas pelo dossel florestal e o período úmido apresentou a maior capacidade de retenção de água, provavelmente pela maior regeneração vegetal nesta época, já que no período seco a queda das folhas pode diminuir a retenção.
\end{abstract}

Palavras-chave: Uso do solo; Interceptação; Impacto

\begin{abstract}
The Atlantic rainforest is one of the most endangered ecosystems of our planet, this area presents only $8 \%$ of its original distribution, needs special attention to improve its conservation goals. This study aimed to compare the water retention capacity of the canopy in two watersheds an entirely covered by Atlantic Forest (Rain Forest), located in "Parque Estadual da Serra do Mar" and other covered with Eucalyptus urophylla x Eucalyptus grandis in an adjacent area. Monitoring was carried out in the years of 2008 and 2009 through rain gauges and the water retention of the forest canopy was evaluated from linear regressions between precipitation (PR) and internal elevation (TR). The results showed that, with the exception of interception in the "dry" season that presented the lowest coefficients of determination (R2), being $77 \%$ in the eucalypt and $82 \%$ in the Atlantic Forest, the variables presented a good fit with the incident precipitation, with higher coefficients of $90 \%$. The Atlantic Forest showed higher retention capacity of water in relation to Eucalyptus, where rainfall lower than $5 \mathrm{~mm}$ in the Atlantic Forest and $0.78 \mathrm{~mm}$ in eucalypt are fully intercepted by the forest canopy and the wet period presented the greatest water retention capacity, probably due to the greater vegetation regeneration at this time, since in the dry period the leaves fall can decrease the retention.
\end{abstract}

Keywords: Land use; Interception; Impact

\footnotetext{
${ }^{\text {I }}$ Engenheiro Ambiental, Dr., Professor da Universidade Vale do Rio Doce, Rua Israel Pinheiro, 2000, Bairro Universitário, CEP 35020-220, Governador Valadares (MG), Brasil. jdgroppo@gmail.com (ORDID: 0000-0002-4820-1632)

II Gestor Ambiental, Dr., Professor da Universidade de Brasília, Campus Planaltina, Área Universitária, 01, Bairro Vila Nossa Senhora de Fátima, CEP 73345-010, Planaltina (DF), Brasil. piposalemi@gmail.com (ORCID: 0000-0003-2271-5712)

III Engenheiro Mecânico, Dr., Professor da Escola de Engenharia de Piracicaba, Av. Monsenhor Martinho Salgot, 560 Bairro Areião, CEP 13414040, Piracicaba (SP), Brasil. jmmoraes066@gmail.com (ORDID: 0000-0001-7945-2381)

${ }^{\text {IV }}$ Engenheiro Ambiental, MSc., Centro de Energia Nuclear na Agricultura, Universidade de São Paulo, Av. Centenário, 303, Bairro São Dimas, CEP 13400-970, Piracicaba (SP), Brasil. digaotrev@yahoo.com.br / gbseghesi@gmail.com (ORDID: 0000-0002-7211-4459)

v Engenheiro Agrônomo, Dr., Centro de Energia Nuclear na Agricultura, Universidade de São Paulo, Av. Centenário, 303, Bairro São Dimas, CEP 13400-970, Piracicaba (SP), Brasil. zebu@cena.usp.br (ORCID: 0000-0002-7103-7551)
} 


\section{Introdução}

O desmatamento é um dos graves problemas que as florestas tropicais vêm enfrentando, gerando consequências locais e até mesmo globais. A Mata Atlântica é um exemplo dessa situação, onde restam poucos fragmentos desse importante bioma no Brasil, sendo que um dos principais remanescentes de Mata Atlântica está localizado na Serra do Mar, na qual foi criado Parque Estadual para proteger esse rico ecossistema.

Esta floresta teve suas riquezas exploradas, e seu espaço cedido para agricultura, criação de gado, construção de centros urbanos e outras atividades, tais como a inserção de florestas plantadas. Esta última pode ser considerada, em certos aspectos, uma alternativa para evitar novos desmatamentos, fornecendo a madeira usualmente retirada das florestas nativas, e quando manejadas corretamente, apresentam uma grande estabilidade no ecossistema, já que os nutrientes introduzidos pela chuva e pelo intemperismo geológico estão em equilíbrio com os nutrientes perdidos para os rios e para o lençol freático (JORDAN, 1982).

Porém, as florestas de crescimento rápido, como é o caso do eucalipto no Brasil, são alvo de muitas controvérsias nos aspectos relacionados às boas práticas de manejo do solo, das águas superficiais e subterrâneas e dos nutrientes, necessitando de um monitoramento contínuo, para garantir a sustentabilidade ecológica e econômica desses ambientes.

Em 1850, na França, foi elaborado um dos primeiros relatos de estudo para verificar os possíveis desequilíbrios que as plantações de eucalipto causam ao ambiente e a partir de então, se propagaram por todo o globo, analisando os aspectos fisiológicos do consumo de água, o balanço hídrico e manejo do solo, em pequenas e grandes escalas, sendo elementos-chave para discussões nos conceitos de sustentabilidade, qualidade e saúde de microbacias. Entretanto, segundo Lima (1996), esses estudos apresentavam geralmente críticas e conclusões negativas a respeito das plantações de eucalipto devido ao fato da espécie ser plantada como monoculturas extensas, deixando o local com baixa diversidade, sendo mais vulneráveis a pragas e mudanças climáticas. $\mathrm{O}$ mesmo autor, em uma vasta revisão bibliográfica, observa ainda que o eucalipto tem comportamento similar ao de qualquer outra espécie florestal, afirmando que os efeitos hidrológicos e biogeoquímicos, em bacias com esse tipo de floresta plantada, podem ser mitigados quando os usuários das mesmas utilizarem práticas de manejo, isto é, práticas que levem ao uso racional desse recurso, visando à sustentabilidade do sistema.

No intuito de alcançar a sustentabilidade na produção florestal, existe a preocupação de desenvolver atividades que não comprometam o equilíbrio no balanço hídrico e de nutrientes do ambiente, mas sim que utilizem o manejo florestal integrado, aliando a parte econômica, social e ambiental (TREVISAN $e t$ al., 2012).

As florestas desempenham papel fundamental na captação e distribuição de água de chuva, assim uma estreita relação com o ciclo hidrológico, interferindo no movimento da água em vários compartimentos do sistema, inclusive nas saídas para a atmosfera e para os rios (ARCOVA; CICCO; ROCHA, 2003).

Em áreas com cobertura florestal parte do aporte da precipitação é interceptada pela copa das árvores antes de atingir o solo e parte da água pluvial é evaporada diretamente para a atmosfera sem haver qualquer interação desta com o solo. A interceptação, segundo Lima (1976) e Tucci e Clarke (1997), é a retenção de parte da precipitação acima da superfície do solo e pode ser calculada subtraindo a precipitação incidente pela precipitação interna mais o escoamento pelo tronco.

A interceptação pode variar muito com o tipo de florestal, pela diferença de fatores como: altura das árvores, tamanho e forma das folhas e densidade da copa (KEIM; SKAUGSET; WEILER, 2005; ZIMMERMANN; ELSENBEER; MORAES, 2006; DE SCHRIJVER et al., 2007; STAELENS et al., 2008; GERMER et al., 2010), além de fatores climáticos (LEVIA JUNIOR; FROST, 2003; CUARTAS et al., 2007; STAELENS et al., 2008; BRAUMAN; FREYBERG; DAILY, 2010).

O objetivo do estudo foi comparar a capacidade de armazenamento de água do dossel vegetativo em uma microbacia coberta por Floresta Ombrófila Densa, (Mata Atlântica), situada no Parque Estadual da Serra do Mar e outra com cobertura de Eucalyptus urophylla x Eucalyptus grandis em área próxima ao Parque. 


\section{Material e Métodos}

Área de estudo

O Núcleo Santa Virgínia é uma área de conservação localizada no interior do Parque Estadual da Serra do Mar, possui uma área de aproximadamente 5.000 hectares de extensão, é coberto predominantemente pela Floresta Ombrófila Densa Montana (VELOSO; RANGEL FILHO; LIMA, 1991), uma vez que o mesmo situa-se a uma altitude que varia de 850 a $1.100 \mathrm{~m}$. Tais florestas são caracterizadas pela ocorrência regular de neblina. Por essa razão, elas podem também ser chamadas de Florestas Nebulares (STADTMULLER, 1987). Cabe salientar que eventos de neblina são constituídos por minúsculas gotas de água que só precipitam quando se deparam com a superfície de objetos sólidos nos quais coalescem para formar gotas maiores que escorrem e precipitam no solo (CHANEY, 1981). Nesta região de escarpas e reversos da Serra do Mar, no Planalto de Paraitinga-Paraibuna, o relevo apresenta fortes declividades. Conforme a classificação Koeppen, o clima regional é tropical temperado, sem estação seca, com precipitação média anual superior a $2.000 \mathrm{~mm}$. A temperatura média anual varia de $22,5^{\circ} \mathrm{C}$ (de $19^{\circ} \mathrm{C}$ no inverno a $25^{\circ} \mathrm{C}$ no verão) na costa até $21^{\circ} \mathrm{C}$ no planalto (TABARELLI; MANTOVANI, 1999).

No interior do Núcleo Santa Virginia encontra-se a microbacia hidrográfica coberta com Mata Atlântica, com 11,5 hectares (ha) de área e 0,28 de declividade média, definida na latitude de $23^{\circ} 19^{\prime} 19^{\prime \prime} \mathrm{S}$ e $45^{\circ} 05^{\prime} 56^{\prime}$ 'W de longitude (Figura 1). A floresta presente na microbacia apresenta de duas a três camadas de copa e também apresenta um sub-bosque de bambu muito denso. A densidade de árvores $(4,8 \mathrm{~cm}$ de diâmetro a altura do peito), estimado em 1.230 indivíduos arbóreos por hectare (ALVES et al., 2010). Os galhos de árvores são frequentemente cobertos por epífitas e musgo durante todo o ano.

A microbacia de eucalipto possui área de 35,5 hectares, sendo 23 hectares de Eucalyptus urophylla x Eucalyptus grandis e 12,5 hectares de APPs e está localizada cerca de $10 \mathrm{~km}$ da microbacia de Mata Atlântica (Figura 1). O plantio foi realizado em 2004, com espaçamento $3 \times 2 \mathrm{~m}$ em talhões e foram respeitadas as áreas de preservação permanente ao longo dos cursos d'água (TREVISAN et al., 2012).

Figura 1 - Área de estudo.

FIgure 1 - Study area.






\section{Metodologia}

O monitoramento foi realizado semanalmente em 2008 e no ano de 2009 passou a ser quinzenal. A precipitação foi obtida com auxílio de um pluviógrafo tipo de báscula (RainLog, RainWise, Inc) instalado em clareiras há aproximadamente $1 \mathrm{~km}$ das microbacias, que registrou a precipitação em intervalos de tempo de 5 minutos.

Para a precipitação que passa pelo dossel da floresta (transprecipitação ou precipitação interna), foram utilizados dez coletores distribuídos aleatoriamente em cada microbacia. $\mathrm{O}$ mesmo número de coletores foi instalado em ambas as microbacias, apesar do eucalipto apresentar área maior, apresenta certa homogeneidade da copa das árvores.

Os coletores foram feitos de PVC com $2 \mathrm{~m}$ de comprimento com uma abertura transversal de 0,15 $\mathrm{m}^{2}$ de área, ligados a um galão, similar ao descrito por Laclau et al. (2003). Informações mais detalhadas estão disponíveis em Groppo (2010).

No presente estudo, a interceptação foi considerada somente a subtração entre a precipitação e a precipitação interna, uma vez que o escoamento pelo tronco é baixo e pode considerado insignificante (LIMA 1976; ARCOVA; CICCO; ROCHA, 2003; LEVIA JUNIOR; FROST, 2003; GOMÉZPERALTA et al., 2008; FREITAS et al., 2013).

A retenção de água do dossel florestal foi avaliada a partir de regressões lineares entre a precipitação (PR) e a precipitação interna (TR). A partir da equação linear, a capacidade máxima de retenção foi obtida considerando o valor da precipitação, quando a precipitação interna igual a zero. Assim, ainda não teve precipitação interna e a totalidade da precipitação é interceptada (CUARTAS et al., 2007; ÁVILA et al., 2014).

\section{Resultados e Discussão}

A precipitação total em 2008 foi de $1.716 \mathrm{~mm}$ na floresta e $1.234 \mathrm{~mm}$ no eucalipto, e em 2009 foi $3.003 \mathrm{~mm}$ na floresta e $1.833 \mathrm{~mm}$ no eucalipto, apresentando alta variabilidade interanual e espacial. Além da diferença na precipitação entre as bacias estudadas, que estão localizadas a poucos quilômetros de distância, a precipitação anual em 2009 foi 70\% e 45\% maiores na floresta e no eucalipto, respectivamente, em comparação com 2008. Houve também uma variação sazonal nas duas microbacias, com aproximadamente $70 \%$ da precipitação anual total ocorrendo no período de verão (outubro a março).

A estação menos úmida ocorreu entre os meses de maio e julho de 2008, com aproximadamente $8 \%$ do total precipitado. Nesse ano, o mês de julho foi o menos chuvoso de todo o período estudado com apenas 4,0 $\mathrm{mm}$. No ano de 2009, todos os meses apresentaram precipitação mais elevada que a do ano anterior, sendo a mínima observada no mês de maio $(74 \mathrm{~mm})$ e a máxima no mês de dezembro (748 $\mathrm{mm})$. Esse último valor representou aproximadamente $25 \%$ do total precipitado do ano de 2009.

Em termos de intensidade de chuva, observou-se que mais de $70 \%$ dos eventos estão na faixa de 0 a $5 \mathrm{~mm} . \mathrm{h}^{-1}$ (Figura 2), e que a contribuição desta classe de intensidade no volume total de chuva foi de $54 \%$ no ano de 2008 e de $36 \%$ no ano de 2009. Já os eventos com intensidades mais altas, ocorrem com menor frequência, se comparados aos de baixa intensidade. Por outro lado, eventos com intensidades superiores a $30 \mathrm{~mm} \cdot \mathrm{h}^{-1}$, apesar de pouco frequentes contribuíram com aproximadamente $12 \%$ do total precipitado no ano de 2008 e $22 \%$ no ano de 2009.

Foi observado o predomínio de chuvas de baixa intensidade $\left(0-5 \mathrm{~mm} \cdot \mathrm{h}^{-1}\right) \mathrm{em}$ ambas as microbacias, porém, a microbacia de Mata Atlântica interceptou o dobro que a microbacia de eucalipto, sendo 30\% do total precipitado enquanto a microbacia de Eucalipto interceptou $14 \%$.

Segundo Bruijnzeel (1990), as florestas tropicais interceptam em média 13\% da precipitação anual. Resultados similares foram encontrados no núcleo de Cunha, também no Parque Estadual da Serra do Mar, em uma bacia coberta com Mata Atlântica (ARCOVA; LIMA; CICCO,1998; ANIDO, 2002; ARCOVA; CICCO; ROCHA; 2003). Na região Amazônica, no estado do Pará, Moraes et al. (2006) encontraram valor em torno de $13 \%$. Próximo a Manaus, no estado do Amazonas, um valor de $20 \%$ foi obtido por Franken et al. (1992) e Leopoldo, Franken e Vila Nova (1995), e no sudoeste do estado de Rondônia, em floresta ombrófila aberta (Terra Firme), a interceptação foi de 11\% (GERMER et al., 2010). 
Figura 2 - Distribuição de frequência de eventos em classes de intensidade de precipitação.

Figure 2 - Frequency distribution of events in classes of precipitation intensity.

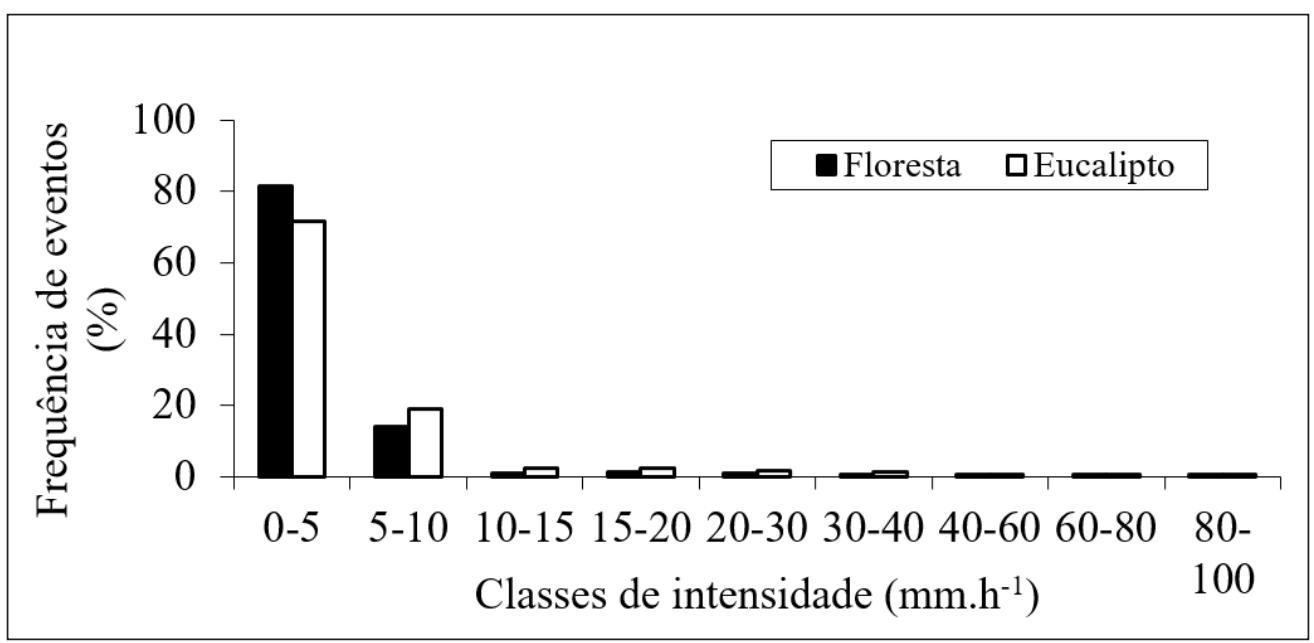

Figura 3 - Regressões lineares: Precipitação Interna X Precipitação; A) Eucalipto; B) Mata Atlântica. Interceptação X Precipitação; C) Eucalipto; D) Mata Atlântica.

Figure 3 - Linear regression: Throughfall X Precipitation; A) Eucalyptus; B) Atlantic Rainforest. Interception X Precipitation; C) Eucalyptus; D) Atlantic Rainforest.
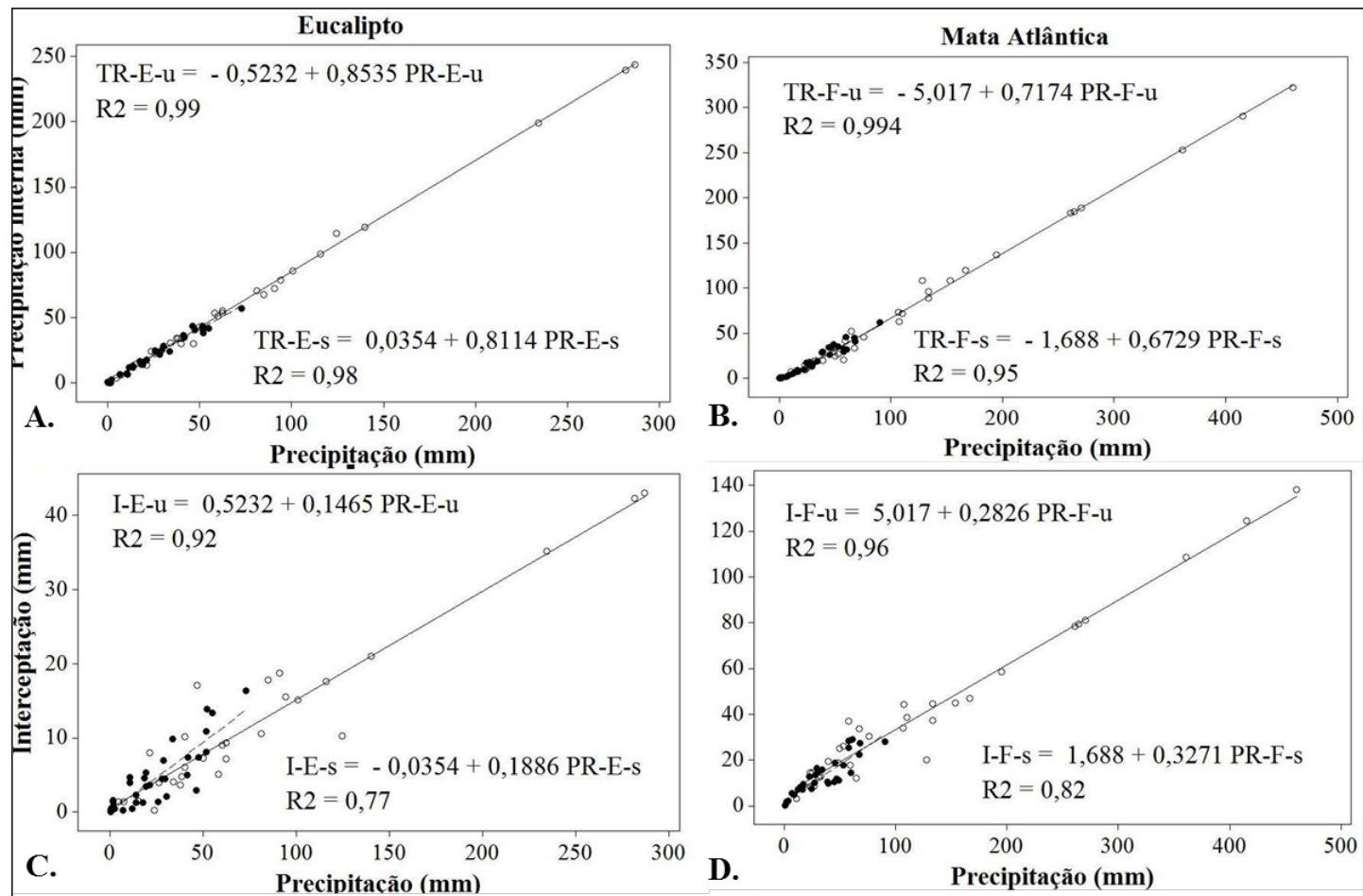

- Período "seco" ○ Período úmido

O valor de interceptação obtido na mata atlântica foi superior aos valores apresentados no parágrafo anterior, porém, é próximo a valores observados em florestas montanas que não sofrem influência significativa da neblina, que interceptam entre $20 \%$ e $50 \%$ da precipitação anual como demonstrado por vários estudos realizados em florestas tropicais (BRUIJNZEEL; HAMILTON, 2000). 
Na Figura 3 são apresentadas as regressões lineares entre a precipitação incidente e a precipitação interna. A série foi dividida em períodos "secos" (abril-setembro) e úmidos (outubro-março).Com exceção da interceptação na época "seca" que apresentou os menores coeficientes de determinação $\left(\mathrm{R}^{2}\right)$, com coeficientes de $77 \%$ no eucalipto e $82 \%$ na Mata Atlântica. As variáveis mostraram bom ajuste com a precipitação incidente com coeficientes maiores 90\%.A Mata Atlântica apresentou maior capacidade de retenção de água das copas em relação ao eucalipto, o que corrobora o alto resultado obtido na interceptação por Groppo (2010), no qual para todo período de estudo foi observado que precipitações inferiores a 5 $\mathrm{mm}$ na Mata Atlântica e $0,78 \mathrm{~mm}$ no Eucalipto são totalmente interceptadas pelo dossel florestal.

Segundo Aston (1979), o índice de área foliar do eucalipto, é tipicamente menor do que o de outras espécies florestais. Portanto, é de se esperar que a sua perda por interceptação seja menor que a das outras espécies.

Pode-se observar também maior capacidade de retenção de água no período úmido em relação ao período seco nos dois tipos de vegetação. Avila et al. (2014) observaram esse mesmo comportamento em uma área coberta com Mata Atlântica na Serra da Mantiqueira, Estado de Minas Gerais. Esses autores atribuíram a maior capacidade de armazenamento de água no período chuvoso na maior regeneração vegetal nesta época, e no período seco está relacionada à menor área do dossel vegetativo proporcionada pela queda das folhas nesta época, ocasionando menor capacidade de armazenamento do dossel da Mata.

Outros estudos realizados tanto em outras áreas de Mata Atlântica como em florestas plantadas são apresentadas na Tabela 1.

Tabela 1 - Resultados em outros estudos.

Table 1 - Results in other studies.

\begin{tabular}{lccc}
\hline & Capacidade retenção (mm) & Cobertura Florestal & Local \\
\hline Presente estudo & 4,85 & Mata Atlântica & Núcleo Santa Virginia - SP \\
& 0,78 & Eucalyptus urograndis & \\
Arcova, Cicco e Rocha (2003) & 0,62 & Mata Atlântica & Núcleo Cunha - SP \\
Moura et al. (2009) & 4,87 & Mata Atlântica & Bacia do Prata - PE \\
Avila et al. (2014) & 1,58 & Mata Atlântica & Serra da Mantiqueira - MG \\
Oliveira Junior e Dias (2005) & 1,3 & Floresta & Estacional \\
& 0,95 & Semidecidual & Agu \\
Lima e Nicolielo (1983) & 0,125 & Pinus caribaea & Pinus oocarpa \\
\hline
\end{tabular}

Os resultados obtidos na área de Mata Atlântica no estudo são bem superiores a outros trabalhos realizados em outras áreas de Mata Atlântica. Arcova, Cicco e Rocha (2003) apesar de ser uma área próxima do presente estudo, encontraram uma capacidade de retenção bem inferior, enquanto Moura et al. (2009), encontraram resultados próximos, em uma bacia de Mata Atlântica no Estado de Pernambuco. Os resultados da microbacia de eucalipto são da mesma ordem de grandeza dos outros trabalhos (LIMA; NICLOLIELO, 1983). As prováveis razões pela alta capacidade de retenção de água observados na microbacia de mata atlântica são a baixa intensidade da chuva e as características da vegetação, como a existência de um dossel de altura média do dossel de 15 metros com algumas árvores emergentes podendo alcançar até 30 metros aliado a um alto índice de área foliar e abundância de epífitas e musgos (ALVES et al., 2010).

\section{Conclusões}

A microbacia de Mata Atlântica interceptou 30\% do total da precipitação incidente, sendo duas vezes mais, comparada à microbacia com cobertura de Eucalyptus urophylla x Eucalyptus grandis. A 
capacidade de retenção de água do dossel também foi maior na Mata Atlântica, provavelmente pela existência de um dossel de altura média do dossel de 15 metros com algumas árvores emergentes podendo alcançar até 30 metros aliado a um alto índice de área foliar e abundância de epífitas e musgos. A maior capacidade de retenção de água observada no período úmido pode estar associada à maior regeneração vegetal nesta época.

\section{Agradecimentos}

À Fundação de Amparo à Pesquisa no Estado de São Paulo - FAPESP, pela bolsa de estudo concedida $n^{\circ} 06 / 51488-0$ e pelo projeto de pesquisa $n^{\circ}$ 06/55136-0. Ao Centro de Energia Nuclear na Agricultura (CENA/USP) e a Escola Superior de Agricultura (ESALQ/USP), por fornecer os meios necessários para a realização desse trabalho.

\section{Referências}

ALVES, L. F. et al. Forest structure and live above ground biomass variation along an elevation gradient of tropical Atlantic moist forest (Brazil). Forest Ecology and Management, Amsterdam, v. 260 , n. 6 , p. $679-91$, jul. 2010.

ANIDO, N. M. R. Caracterização hidrológica de uma microbacia experimental visando identificar indicadores de monitoramento ambiental. 2002. 69 f. Dissertação (Mestrado em Recursos Florestais) Escola Superior de Agricultura Luiz de Queiroz, Universidade de São Paulo, Piracicaba, 2002.

ARCOVA, F. C. S.; CICCO, V.; ROCHA, P. A. B. Precipitação efetiva e interceptação das chuvas por floresta de Mata Atlântica em uma microbacia experimental em Cunha - São Paulo. Revista Árvore, Viçosa, MG, v. 27, p. 257-262, mar./abr. 2003.

ARCOVA, F. C. S.; LIMA, W. P.; CICCO, V. Balanço hídrico de duas microbacias hidrográficas no Laboratório de Hidrologia Florestal Walter Emmerich, São Paulo. Revista Instituto Florestal, São Paulo, v. 10, n. 1, p. $39-51,1998$.

ASTON, A. R. Rainfall interception by eight small trees. Journal Hydrology, Amsterdam, v. 42, n. 3/4, p. 383-396, jul. 1979.

AVILA, L. F. et al. Participação da precipitação pluvial em um microbacia hidrográfica ocupada por mata atlântica na Serra da Mantiqueira. Ciência Florestal, Santa Maria, v. 24, n. 3, p. 583-595, jul./set. 2014.

BRAUMAN, K. A.; FREYBERG, D. L.; DAILY, G. C. Forest structure influences on rainfall partitioning and cloud interception: a comparison of native forest sites in Kona, Hawai'i. Agricultural and Forest Meteorology, New Yorke, v. 150, n. 2, p. 265-275, fev. 2010.

BRUIJNZEEL, L. A. Hydrology of moist tropical forests and effects of conversion: a state of knowledge review. Paris: UNESCO; IHP, 1990. 224 p.

BRUIJNZEEL, L. A.; HAMILTON, L. S. Decision time for cloud forests. Paris: UNESCO; IHP, 2000.

CHANEY, W. R. Sources of water. In: KOSLOWSKI, T. T. (Ed.). Water deficits and plant growth. VI. Woody plant communities. New York: Academic Press, 1981. p. 1-47.

CUARTAS, L. A. et al. Interception water-partitioning dynamics for a pristine rainforest in Central Amazonia: marked differences between normal and dry years. Agricultural and Forest Meteorology, Amsterdam, v. 145, n. 1/2, p. 69-83, jul. 2007.

DE SCHRIJVER, A. et al. The effect of forest type on throughfall deposition and seepage flux: a review. Oecologia, Heidelberg, v. 153, n. 3, p. 663-674, set. 2007.

FREITAS, J. P. O. et al. Distribuição da água de chuva em Mata Atlântica. Revista Ambiente \& Água, Taubaté, v. 8, n. 2, p. 100-108, abr. 2013. 
FRANKEN, W. et al. Estudo da interceptação da água de chuva em cobertura florestal amazônica do tipo terra firme. Acta Amazônica, Manaus, v. 12, n. 2, p. 327-331, abr./jun. 1992.

GERMER, S. et al. Influence of land-use change on near-surface hydrological processes: Undisturbed forest to pasture. Journal of Hydrology, Amsterdam, v. 380, n. 3/4, p. 473-480, jan. 2010.

GROPPO, J. D. Caracterização hidrológica e dinâmica do nitrogênio em uma microbacia com cobertura florestal (Mata Atlântica), no Parque Estadual da Serra do Mar, Núcleo Santa Virgínia. 2010. 81 f. Tese (Doutorado em Ciências) - Centro de Energia Nuclear na Agricultura, Universidade de São Paulo, Piracicaba, 2010.

JORDAN, C. F. The nutrient balance of amazonian rain forest. Ecology, Brooklyn, v. 63, n. 3, p. 647$654,1982$.

KEIM, R. F.; SKAUGSET, A. E.; WEILER, M. Temporal persistence of spatial patterns in throughfall. Journal of Hydrology, Amsterdam, v. 314, n. 1-4, p. 263-274, nov. 2005.

LACLAU, J. P. et al. Nutrient cycling in a clonal stand of Eucalyptus and adjacent savanna ecosystem in congo.1 Chemical composition rainfall, throughfall and stemflow solutions. Forest Ecology and Management, Oxford, v. 176, n. 1/3, p. 527-577, mar. 2003.

LEOPOLDO, P. R.; FRANKEN, W. K.; VILA NOVA, N. A. Real evapotranspiration and transpiration through a tropical rain forest in central Amazônia as estimated by the water balance method. Forest Ecology and Management, Amsterdam, v. 73, n. 1/3, p. 185-195, may 1995.

LEVIA JUNIOR, D. F.; FORST, E. E. A review and evaluation of stemflow literature in the hydrologic and biogeochemical cycles of forested and agricultural ecosystems. Journal of Hydrology, Amsterdam, v. 274 , n. 1/4, p. 1-29, abr. 2003.

LIMA, W. P. Impacto ambiental do eucalipto. 2. ed. São Paulo: EDUSP, 1996. 301 p.

LIMA, W. P. Interceptação da chuva por povoamentos de eucaliptos e de pinheiros. Revista IPEF, Piracicaba, v. 13, p. 75-90, 1976.

LIMA, W. P.; NICOLIELO, N. Precipitação efetiva e a interceptação em florestas de Pinheiros tropicais e em uma reserva de Cerradão. Revista IPEF, Piracicaba, n. 24, p. 43-46, 1983.

MORAES, J. M. et al. Water storage and runoff processes in plinthic soils under forest and pasture in Eastern Amazonia. Hydrological Processes, Chichester, v. 20, n. 12, p. 2509-2526, jul. 2006.

MOURA, A. E. S. S. et al. Interceptação das chuvas em um fragmento de floresta da Mata Atlântica na Bacia do Prata, Recife, PE. Revista Árvore, Viçosa, MG, v. 33 n. 3, p. 461-469, maio/jun. 2009.

OLIVEIRA JÚNIOR, J. C.; DIAS, H. C. T. Precipitação efetiva em fragmento secundário da mata atlântica. Revista Árvore, Viçosa, MG, v. 29, n. 1, p. 9-15, jan./fev. 2005.

STADTMULLER, T. Cloud forests in the humid tropics: a bibliographic review. Tokyo: The United Nations University, 1987. 81 p.

STAELENS, J. et al. Rainfall partitioning into throughfall, stemflow, and interception within a single beech (Fagus sylvatica L.) canopy: influence of foliation, rain event characteristics, and meteorology. Hydrological Processes, New York, v. 22, n. 1, p. 33-45, maio 2008.

TABARELLI, M.; MANTOVANI, W. A regeneração de uma floresta tropical montana após corte e queima (São Paulo - Brasil). Revista Brasileira de Biologia, São Carlos, v. 59, n. 2, p. 239-250, 1999.

TREVISAN, R. et al. Dinâmica da água em uma microbacia florestal de eucalipto localizada na Serra do Mar no Vale do Paraíba do Sul. Revista Brasileira de Recursos Hídricos, Porto Alegre, v. 17, n. 4, p. 207-216, out./dez. 2012.

TUCCI, C. E. M.; CLARKE, R. T. Impacto das mudanças da cobertura vegetal no escoamento: revisão. Revista Brasileira de Recursos Hídricos, Rio de Janeiro, v. 2, n. 1, p. 135-152, jan./jul. 1997. 
VELOSO, H. P.; RANGEL FILHO, A. L. R.; LIMA, J. C. A. Classificação da vegetação brasileira, adaptada a um sistema universal. Rio de Janeiro: IBGE, Departamento de Recursos Naturais e Estudos Ambientais, 1991.

ZIMMERMANN, B.; ELSENBEER, H.; MORAES, J. M. The influence of land-use changes on soil hydraulic properties: implications for runoff generation. Forest Ecology and Management, Amsterdam, v. 222, n. 1-3, p. 29-38, fev. 2006. 